\title{
ANTONIO BERAMENDI Y FREYRE. UN DIPLOMÁTICO EN LOS INICIOS DEL SIGLO XIX
}

\author{
Emilio SOLER PASCUAL
}

\section{Antecedentes familiares}

El ayuntamiento de Beramendi, en el antiguo Reino de Navarra, es el origen del apellido de la familia Beramendi ${ }^{1}$.

Las diversas ramas de los Beramendi ${ }^{2}$ se extendieron por toda Navarra, pero fue la familia nacida del matrimonio de Simón de Beramendi con María de Huarte la que originó, años después, el nacimiento de Antonio Beramendi y Freyre.

En 1771, el fiscal del Real Consejo del Reino de Navarra entabló pleito contra la familia de Manuel Beramendi, abuelo de Antonio Beramendi, acusándoles de uso indebido de escudo nobiliario ${ }^{3}$. La causa fue ganada, tras muchos meses, por la familia Beramendi que adquirió, de forma oficial, el certificado de su hidalguía. Los informes, contrainformes, declaraciones y atestados de este juicio pueden estudiarse en el Archivo Histórico Nacional ${ }^{4}$, expediente abierto a D. Pantaleón de Beramendi, padre de Antonio Beramendi, para la obtención del título de Caballero de la Orden de Carlos III.

Los padres de Antonio fueron Pantaleón de Beramendi y Cecilia Freyre. Pantaleón de Beramendi y de Ibiricu nació en Pamplona el 28 de julio de 1746 y fue bautizado en la iglesia de San Saturnino5. Perteneció al Consejo de S.M., siendo su Secretario de Decretos; fue oficial segundo de la Secretaría del Despacho Universal de la Real Hacienda. Fue nombrado Regidor Perpetuo de la Villa de Madrid y, más tarde, ministro de la Real Junta de Comercio, Moneda y Minas ${ }^{6}$. Por Real Decreto de 13 de enero de 1789, fue nombrado Caballero de la Orden de Carlos III 7 .

Cecilia Freyre, madre de Antonio Beramendi, había nacido en Alicante el 8 de marzo de $1750^{8}$. Cecilia fue bautizada en la iglesia parroquial de San Nicolás "como hija lexítima del capitán del regimiento de infantería de Zamora Don Francisco 
Freyre, natural de Amberes, en Flandes, y de Dña. Pasquala de Bruín, natural de la ciudad de Valencia9. Pantaleón y Cecilia contrajeron matrimonio en Madrid el $15 \mathrm{de}$ marzo de $1772^{10}$, en la Real Parroquia de la Real Capilla de S.M ${ }^{11}$.

Antonio Beramendi y Freyre nació en Madrid el 21 de octubre de 1777 y fue bautizado, un día después, en la parroquia de la Santa Cruz de Madrid ya que sus padres vivían cerca de allí, en la calle de Carretas ${ }^{12}$. Antonio fue bautizado por D. Gil Guerrero, teniente cura de dicha iglesia, con los nombres de Antonio, Buenaventura, Hylarion, Alfonso y tuvo como padrino de pila a D. Alfonso Cervantes, parroquiano de San Sebastián ${ }^{13}$ y del Consejo de S.M.14, secretario de Estado del Despacho Universal de Hacienda de España e Indias como Carlos Beramendi, su hermano.

Hermano mayor de Antonio fue Carlos Beramendi, intendente de ejército ${ }^{15}$ y cónsul de España en los Países Bajos ${ }^{16}$ durante el Trienio Liberal. Carlos Beramendi nació en Pamplona ${ }^{17}$ (como su padre, su abuelo y su bisabuelo) entre los años 1773 y 1776.

\section{Roma y la Orden de Carlos III}

Antonio Beramendi y Freyre se instruyó en la latinidad y pasó a la Universidad de Salamanca ${ }^{18}$. Allí estudió Filosofía y Moral "con aplicación y particular aprovechamiento, y sus Exercícios le fueron aprobados nemine discrepante". En Salamanca se graduó de Bachiller en Filosofía. Posteriormente se dedicó a las Humanidades, y ganó dos matrículas en la Cátedra de su enseñanza, "acreditando su talento y aplicación". Más tarde, estudió durante un año Instituciones Civiles en la Universidad de Salamanca, "que incorporó en la de Alcalá, y habiéndose matriculado en ésta en tiempo y debida forma continuó ganando y probando el segundo de Lcyes, como también el primero y segundo de Instituciones Canónicas, y tercero y quarto de Jurisprudencia."

En 6 de junio de 1796 recibió el Grado de Bachiller en Cánones ${ }^{19}$, aprobando "los Exercícios prevenidos en la Real Cédula del año mil setecientos setenta, que desempeñó con mucho lucimiento, y le fueron aprobados, también, nemine discrepante."

Asimismo, "habiendo practicado, según dicha Real Cédula, los correspondientes Excrcícios, que desempeñó con el propio lucimiento de los anteriores, y le fueron aprobados en iguales términos", recibió el Grado de Bachiller en Leyes el día doce de junio de 1796. El cinco de mayo de 1799 recibió el Grado de Licenciado en ambos Derechos, y el de Doctor "con la pompa y solemnidades prevenidas en las Reales Constituciones de la expresada Universidad", cuatro días después ${ }^{20}$.

Antonio Beramendi, que perteneció a la Academia de Santa María de Jesús de la citada Universidad, sustituyó varias veces a los titulares de las Cátedras de Leyes y Cánones por nombramiento del Rector.

Antonio Beramendi fue nombrado agregado a la Secretaría del Ministerio a la Corte de Roma por Real Orden de 4 de enero de $1801^{21}$, con un sueldo anual de 
12.000 reales $^{22}$. Además de su impresionante historial académico debió de influir la brillante carrera administrativa de su padre, D. Pantaleón y el que su tío hubicra sido el Ilmo. Señor D. Fray Joaquín de Eleta, "Arzobispo y Obispo de Osma", y que fue confesor de S.M. Carlos III y de S.M. Carlos IV e "Inquisidor de la Suprema"25.

Antonio Beramendi, casado con María de la Concepción Goicolea ${ }^{26}$, tuvo un hijo, Francisco de Beramendi y Goicolea, que fue Oficial Mayor de la Secretaría Particular de S.M ${ }^{27}$. a propuesta de D. Guillermo Morphy, secretario particular de S.M. ${ }^{28}$ Francisco Beramendi fue Caballero de la Orden de San Juan y Caballero de San Mauricio. ${ }^{29}$

Antonio Beramendi y Freyre, siendo agregado a la Secretaría de Legación en la Corte de Roma fue nombrado Caballero de la Real Orden española de Carlos III por Real Decreto de 24 de octubre de $1806^{30}$. Las pruebas por las que tuvo que pasar su expediente fueron aprobadas en 17 de marzo de 1808 y tuvo como testigos de excepción a personajes muy importantes de la Corte $^{31}$.

El motivo de la concesión de la condecoración lo describe él mismo:32

"Desde mi llegada a Roma fuí encargado por mi Gefe del Despacho de todos los asuntos de la Legación de Etruria unida entonces a aquel Ministerio. En el año 1806 fuí comisionado para conducir a Madrid unos pliegos importantes del Ministerio de Nápoles para el Gobierno lo que ejeculé cerca de su satisfacción que me fue remunerado con la gracia de la Cruz de la Real Orden de Carlos III y por no haber vacante de las pensionadas se me concedió la correspondiente sobre una de las Dignidades reservadas".

También desde Roma, el 28 de diciembre de 1807, se certificó la buena conducta de Antonio Beramendi para que pudiese ser poseedor de la Orden de Carlos III. Tanto el Notario Apostólico, D. Sebastián de Navasques, como el Secretario del Ministerio de S.M. Católica, D. Francisco Xavier de Elexaga, en nombre del Excmo. Sr. D. Antonio de Vargas y Laguna, ministro plenipotenciario de España en Roma así lo efectuaron ${ }^{33}$.

El profesor Mercader Riba ${ }^{34}$ señala que "el ministro ordinario español en Roma, Antonio Vargas Laguna, había sido arrestado por el gencral francés ocupantc, conde Miollis, juntamente con su secretario, Antonio Beramendi y Freyre, y otros agregados o funcionarios de la propia legación, por haberse negado a prestar el juramento de fidelidad y obediencia a José Bonaparte".

El propio Antonio Beramendi relata en su expediente del Ministerio de Asuntos Exteriores ${ }^{35}$ este suceso:

“... En el año de 1808 fuí arrestado en Roma por Orden del Emperador Napoleón, por no haber querido prestar el juramento de reconocimiento de su hermano José Bonaparte como Rey de España y conducido al castillo de Fenestrelle cn los Alpes en donde permanecí cinco años."

Antonio Beramendi seguía los pasos de su hermano Carlos que fue un destacado luchador anti-bonapartista y que fue condecorado y herido, varias veces, durante 
la guerra de la Independencia ${ }^{37}$. Contrastaba esta actitud de sus hijos con la de D. Pantaleón de Beramendi y Eleta, que con arreglo al Decreto de 16 de febrero de 1809, juraba lealtad a José $\mathrm{I}^{38}$.

Una vez arrestado Antonio Beramendi, el ministro de Negocios Extranjeros francés y considerando que "sería peligroso dejar en Roma mismo a estos sugetos condecorados, cuya situación inspira vivo interés y en donde hay hasta ciertos motivos de disgusto y agitación" aconseja a su soberano que ordene al general Miollis que haga llevar a Francia al ministro Vargas Laguna y a su secretario Antonio Beramendi con cierto decoro, a disposición del emperador, ya que se hallan también allí detenidos otros sujetos, por aquel mismo motivo, y no convendría tampoco trasladarlos a Florencia, ni mucho menos hacerles llegar a España ${ }^{39}$.

La negativa a prestar el juramento de fidelidad bonapartista le supuso a Antonio Beramendi el ser encerrado durante cinco años en varias prisiones francesas, de las que sólo salió cuando acabó la guerra de la Independencia. Se le condecoró con la medalla de los Prisioneros de Estado en Castillo y con la de los Civiles, en el tiempo de la guerra de la Independencia ${ }^{40}$.

En 1813, cuando entraron las tropas de los Aliados en Italia se le trasladó al Castillo de Pierre-Chate ${ }^{41}$ en Saboya y no creyéndolos todavía seguros, transportaron a todos los que allí había al castillo de Saumur ${ }^{-42}$, en el que permaneció Antonio Beramendi hasta fines de abril de 1814. Como hemos visto en la nota 42, el profesor Aymes que en su libro sobre La deportación bajo el Primer Imperio no cita a Antonio Beramendi como deportado español, aquí lo menciona como "algunos otros difíciles de identificar" y le llama Beramundi. En aquella época, habiendo entrado las tropas aliadas en París, se le puso en libertad después de cinco años y medio de cautiverio 43 .

\section{Cónsul en Génova}

En el expediente del Archivo de Asuntos Exteriores sobre Antonio Beramendi se encuentra una anotación titulada: Beramendi, $1-1 /$ en el que se cita a Don Antonio de Beramendi y Freyre Cónsul General de Génova y se dice textualmente:

"Este sujeto después de haber concluído su carrera literaria, lue destinado a Roma en 1804 de Agregado a aquella Legación. En 1809 fue encerrado en una de aquellas fortalezas por no haber querido prestar a jurar al Gobierno intruso de España. Puesto en libertad en 1814 y restituído a esta Corte, se pensó en darle plaza en esta Secretaría en atención a sus méritos y circunstancias, pero prelïrió ir de Cónsul General a Génova, cuyo destino era entonces muy lucrativo y de más representación". El sueldo era de 30.000 reales anuales ${ }^{45}$.

El 19 de junio de 1814 un Real Decreto nombró a Antonio Beramendi Encargado de Negocios y Comercio cerca de la República de Génova "y por haver dejado de existir ésta y agregado su territorio al Rey de Cerdeña por el Tratado de Viena, se le declaró Cónsul General en dicho puerto de Génova y sus dependencias 
por Real Decreto de 1 de Noviembre de 1815". En este puesto Antonio Beramendi sustituyó a Antonio López Gonzalo ${ }^{46}$.

Nada más llegar a su nuevo destino en Génova, Antonio Beramendi comenzó a inventariar los archivos de aquel Consulado "que se hallaban en entero desorden" y que "mereció la aprobación del Gobierno"47.

En 1817, Antonio Beramendi se desplazó a Turín como Secretario de la Comisión que tuvo D. Eusebio Bardají y Azara, embajador en la Corte de Turín, para tomar posesión de los Estados de Luca a nombre de S.M. la Reina Dña. María Luisa de Borbón en Reina de Etruria ${ }^{48}$. Antonio Beramendi permaneció todavía algunos meses en la Corte de Turín como Encargado de Negocios hasta que el Gobierno espanol nombró un Ministro residente en dicha Corte ${ }^{49}$.

El uno de septiembre de 1823 la Regencia del Reino, por Decreto del 31 de agosto, lo declaró cesante.

El motivo de su cese como Cónsul en Génova lo aclara, en parte, el documento existente en el Archivo del Ministerio de Asuntos Exteriores ${ }^{50} \mathrm{en} \mathrm{el} \mathrm{que} \mathrm{por} \mathrm{un}$ expediente de 31 de agosto de 1823, S.M. resuelve que D. Antonio Beramendi sca separado de su destino en el Puerto de Génova por sospechoso: "El Conde de Sambruy, encargado de negocios de S.M. el Rey de Cerdeña (...) dice ha sido informado (...) recelar de la conducta de D. Antonio Beramendi, Cónsul General de S.M. en Génova (...) el referido Beramendi mantiene una Correspondencia secreta con los Principales refugiados Piamonteses y diferentes sectarios de la Italia por medio del Cónsul de S.M. en Gibraltar (...) que se separe del destino de Cónsul de España al mencionado Beramendi y se obtenga aquella pronta satisfacción que pide este asunto...".

Parece cvidente que el liberalismo consustancial a los dos hermanos Beramendi, Carlos y Antonio, llevó a éste a tener que permanecer cinco años cesante en espera de mejores vientos políticos que le llevaran a otro destino diplomático.

El profesor Gil Novales, en su Diccionario biográfico del Trienio Liberal5!, señala que tras el cese de Antonio Beramendi en su destino de Génova, se desplazó a París en octubre de 182352 y volvió a Madrid el 15 de enero de 1824.

Carlos Beramendi, hermano de Antonio, describe en su opúsculo La inocencia triunfa al fin de la calumnia ${ }^{53}$ que cuando se decide a pedir una licencia temporal para salir de España, donde la Inquisición seguía sus pasos cada vez más cerca ${ }^{54}$, elige partir a Italia donde su hermano Antonio y su hijo Carlos eran Cónsul y vice-cónsul, respectivamente.

En la Biblioteca Nacional, la Guía de Forasteros en Madrid55, en sus diversas anualidades nos testimonian la presencia de Antonio Beramendi y Freyre como Cónsul General en Génova. Así figura en la Guía de 1815, en la de 1816, 1817, 1818, 1819 y 1820. También aparece el nombre de Carlos Beramendi Galney [por Galwey $]^{56}$ durante las Guías de $1816,1817,1818,1819$ y 1820 ejerciendo el cargo de vice-cónsul en Génova. 


\section{El consulado general de Marruecos}

El 31 de mayo de 1828, el Rey, "por su Real Decreto de dicho día", nombraba a Antonio Beramendi Cónsul General y Encargado de Negocios de S.M. en Marruecos con residencia en Tánger, con un sueldo anual de 60.000 reales y 40.000 más para gastos 57 .

La primera misión que se le encomendó en Tánger fue la de arreglar dos antiguas reclamaciones pendientes entre los gobiernos de Marruecos y España: "la una sobre una presa de consideración y la otra sobre las deudas que contrajo aquí D. Zenón de Ozué, cuyas dos reclamaciones aunque ascendían a más de 34.000 duros, las he arreglado por 12.000 comprendido todo gasto, por cuyo motivo recibí entonces la más completa aprobación del Gobierno"58.

Casi al mismo tiempo de tomar posesión de su cargo de Embajador, Antonio Beramendi contribuyó, a invitación del Gobierno, a socorrer a los habitantes de Torrevicja y sus alrededores por la desgracia que les supuso el terremoto de dicho año 59 .

E1 27 de abril de 1829, y desde Madrid, Antonio Beramendi escribe ${ }^{60}$ al ministro Manuel González Salmón interesándose por la solución a los problemas que tiene planteados con la Real Hacienda su hermano Carlos, que "implora de S.M. una jubilación o retiro, en atención a sus méritos y años de servicio...". Poco podía sospechar Antonio Beramendi que los problemas de cobro de pensión de jubilación que tuvo su hermano Carlos hasta su muerte en $1832^{61}$, volverían a repetirse, casi punto por punto, con su propia jubilación como Baile General de Valencia ${ }^{62}$.

En el Archivo Histórico Nacional ${ }^{63}$, sección de Estado, se encuentra depositada la correspondencia mantenida por D. Antonio Beramendi con la Secretaría de Estado durante los años comprendidos entre 1829 y 1831 .

Posteriormente, Antonio Beramendi se encargó de la negociación del reconocimiento del antiguo Tratado entre los Gobiernos de Nápoles y el de Marruecos que concluyó satisfactoriamente a tenor de las instrucciones que se le dieron, y aunque no tuvo efecto lo estipulado fue, en palabras de Antonio Beramendi, "por recader el Gobierno de Nápoles de lo que había prometido, pues el Gobierno de Marruecos ha aprobado enteramente cuanto ha ejecutado"64.

En febrero de 1834, Antonio Beramendi se encontraba en Madrid ya que su esposa se encontraba en avanzado estado de gestación. Su suegra, Dña. Josefa Ariza de Valenzuela, Dueña de S.M. "con destino al Quarto de la Serenísima Señora Infanta Dña. Luisa Carlota", escribió a S.M., en 27 de lebrero de 1834, para "que S.M. tuviese a bien concederla el que su yerno D. Antonio Beramendi pudiese continuar en esta Corte hasta que su esposa, hija de la esponente, saliese de su cuidado, mediante lo adelantado que se encontraba en su embarazo..."65.

La correspondencia mantenida por Antonio Beramendi con la Secretaría de Estado por asuntos derivados de su responsabilidad y durante el período 1834-1840 se encuentra depositada en el Archivo Histórico Nacional66. 
En 1835, señalaba Antonio Beramendi67 que el "24 de Octubre de 1835 he hecho donación del $15 \%$ de mi sueldo mientras duren las actuales circunstancias de la guerra."

En 1836, Antonio Beramendi realizó, por escrito, el juramento de fidelidad a la Reina y prometió observar la Constitución política de la Monarquía española decretada por las Cortes en 1812. Él mismo nos lo cuenta:

"Lo he prestado el 19 del corriente con arreglo al Real Decreto de 13 de Agosto de este año y a las Reales Ordenes de 23 y 30 de setiembre..."68.

Además, Antonio Beramendi declara voluntaria y libremente que:

"mis opiniones políticas como Cónsul General y Encargado de Negocios de S.M. han sido y serán siempre conformes a los principios ya sea de la Constitución política mandada observar o a los que la Representación Nacional en unión con el Gobierno de S.M. de quien dependo quisieran establecer pues mi divisa ha sido siempre la de obedecer y no contrariar el orden establecido; y mis sentimientos respecto a la sagrada persona de la Reyna de las Españas Doña Isabel II y sus legítimos dercchos a la Corona los más sinceros y leales". Tanger veinte de Noviembre de 1836. Firmado y rubricado Antonio de Beramendi y Freyre ${ }^{69}$.

La carrera diplomática de Antonio Beramendi pudo haber fïnalizado con el cese de su actividad en Tánger en 184070. Un Beramendi probablemente cansado de estar alejado de su familia, tras 40 años de carrera diplomática, aceptó el nombramiento de Gentilhombre de Cámara. El 17 de octubre de 1840 se produjo el Rcal Decreto $^{71}$ :

"Palacio, 17 de octubre de 1840. A Don Antonio de Beramendi, Cónsul General de Tánger y Encargado de Negocios en el Reyno de Marruccos. Excelencia= S.M. por Decreto de este día se ha dignado conceder a V.E. la Llave de Gentilhombre de Cámara con ejercício, libre de gastos. Lo que Real Orden comunico a V.E. para su inteligencia y satisfacción...".

Antonio Beramendi y Freyre juró el cargo de Gentilhombre de Cámara en ejercicio, en Palacio el 7 de mayo de 1841, en manos del Exmo. Sr. Duque de Híjar, Sumiller de Corps de S.M. y ante Don Joaquín de Borjas Tarrius, secretario de S.M. la Reina ${ }^{72}$. 


\section{NOTAS}

1.- GONZÁLEZ DORIA, Fernando: Diccionario Heráldico y Nobiliario de los Reinos de España. Ed. Bitácora. Madrid, 1897. pp. 124: “... Del lugar de su nombre, ayuntamiento de Basaburúa Mayor, partido judicial de Pamplona."

2.- GARCÍA CARRAFFA, Alberto y Arturo: Enciclopedia Heráldica y Genealógica Hispano-Americana. Madrid, Imprenta de Antonio Marzo, 1924. Tomo XVI. BENCBLAN. pp. 41: “... En Navarra, con solar y palacio, en el lugar de Beramendi (cuyo nombre tomó), del Ayuntamiento de Basaburúa Mayor, y partido judicial de Pamplona. Ramas de este solar radicaron en la ciudad de Pamplona; en la villa de Mendigorria, del partido judicial de Tafalla; en la villa de Mendovia, del partido judicial de Estella; en el lugar de Muzquiz, del partido judicial de Pamplona; y en el de Aizcorbe, del mismo partido..."

3.- GARCÍA CARRAFFA, Alberto y Arturo: Enciclopedia Heráldica y Genealógica Hispano-Americana. Op. cit. pp. 42: "ARMAS.- El Palacio del lugar de Beramendi traía en su escudo dos osos, uno sobre otro. Así consta, pero sin indicar sus esmaltes, en documentos de la nobleza ejecutoriada de Navarra."

4.- AHN. Estado. Orden de Carlos III. Expediente 320. Pruebas de D. Pantaleón de Beramendi.

5.- AHN. Estado. Orden de Carlos III. Expediente 320. Pruebas de D. Pantalcón de Beramendi.

6.- AHN. Estado. Orden de Carlos III. Expediente 1379. Pruebas de D. Antonio Beramendi y Freyre.

7.- AHN. Estado. Orden de Carlos III. Expediente 320. Pruebas de D. Pantaleón de Beramendi.

8.- AHN. Estado. Orden de Carlos III. Expediente 320. Pruebas de D. Pantaleón de Beramendi.

9.- AHN. Estado. Orden de Carlos IIl. Expediente 1379. Pruebas de D. Antonio Beramendi y Freyre.

10.- AHN. Estado. Orden de Carlos III. Expediente 320. Pruebas de D. Pantalcón de Beramendi.

11.- AHN. Estado. Orden de Carlos III. Expediente 1379. Pruebas de D. Antonio Beramendi.

12.- AHN. Estado. Orden de Carlos III. Expediente 1379. Pruebas de D. Antonio Beramendi.

13.- AHN. Estado. Orden de Carlos III. Expediente 1379. Pruebas de D. Antonio Beramendi y Freyre.

14.- AHN. Estado. Orden de Carlos III. Expediente 1379. Pruebas de D. Antonio Beramendi.

15.- SOLER PASCUAL, Emilio: Viajes y acción política del intendente Carlos Beramendi. Tesis doctoral inédita. Universidad de Alicante, junio de 1993.

16.- AMAE. Personal español. Legajo 25. Número 1085. Expediente de D. Carlos Beramendi.

17.- AHPM. Legajo 4310. Folio 21 y ss. Testamento de D. Ricardo Galvey y de Dña. Margarita Beramendi.

18.- AMAE. Personal español. Legajo 23. Número 1051. Expediente de D. Antonio Beramendi.

19.- AMAE. Personal español. Legajo 23. Número 1051. Expediente de D. Antonio Beramendi. 
20.- AMAE. Personal español. Legajo 23. Número 1051. Expediente de D. Antonio Beramendi.

21.- AMAE. Personal español. Legajo 23. Número 1051. Expediente de D. Antonio Beramendi.

22.- AMAE. Personal español. Legajo 23. Número 1051. Expediente de D. Antonio Beramendi.

23.- LOPERRÁEZ CORVALÁN, Juan: Descripción histórica del Obispado de Osma. Madrid, en la Imprenta Real, 1788.

24.- VILLANUEVA, Joaquín Lorenzo: Vida literaria de D. Joaquín Lorenzo Villanueva o Memoria de sus escritos y de sus opiniones eclesiásticas y políticas, y de algunos sucesos notables de su tiempo. 2 vls. Londres. En la Imprenta de A. Macintosh, 20, Great New Street. MDCCCXXV. Tomo Il cap. VIII pp. 67: "Carlos IV siendo Príncipe de Asturias tuvo por confesor algunos años al Padre Eleta: luego por inllujo del Conde de Floridablanca eligió para este ministerio a su paisano y amigo don Allonso Camacho..."

25.- AMAE. Personal español. Legajo 23. Número 1051. Expediente de D. Antonio Beramendi.

26.- AHN. Estado. Orden de Carlos III. Expediente 1379. Apéndice: pruebas de su mujer.

27.- AGP. Expedientes personales. Caja 117. Expediente 27: "Don Francisco Beramendi: Por Real Decreto de 18 de enero de 1875 fue nombrado Oficial Mayor de la Secretaría Particular de S.M. con el sueldo anual de seis mil quinientas pesetas anuales scñalado cn la plantilla aprobada en 17 de enero."

28.- AGP. Expedientes personales. Caja 117. Expediente 27: El 17 de enero de 1875 D. Guillermo Morphy, secretario particular de S.M., propone a D. Francisco de Beramendi y Goicolea, jele de Administración cesante, al Intendente General de Ia Real Casa y Patrimonio para el cargo de Oficial Mayor. Ambos documentos fucron ratificados por S.M. al día siguiente. Otro documento que consta es el del nombramiento con la firma del Rey. Francisco Beramendi prestó juramento (consta el acta de juramento al soberano Alfonso XII). El doce de agosto de 1880 cesó como Oficial Mayor de la Secretaría.

Existe una acotación posterior que señala que: “... en agosto de 1880 pasó al servicio del Estado a las Islas Filipinas."

29.- AMAE. Personal español. Legajo 30. Número 1194. Expediente de D. Francisco Beramendi y Goicolea.

30.- AHN. Estado. Orden de Carlos III. Expediente 1379. Pruebas de D. Antonio Beramendi y Freyrc.

31.- AHN. Estado. Orden de Carlos III. Expediente 1379. Pruebas de D. Antonio Beramendi y Freyre: Fueron testigos de las pruebas:

- El Exmo. Señor D. Patricio Martínez de Bustos, Arcediano de Trastámara, Dignidad, y Canónigo de la Santa Iglesia de Santiago. Caballero Gran Cruz de la Real y distinguida Orden Española de Carlos III. Individuo nato de la Real Junta de la Inmaculada Concepción. Exactor y Colector de las Pensiones consignadas a la misma Real Orden. Juez privativo del nuevo Rezado, del Consejo de S.M. y Comisario Apostólico General de las tres gracias de Cruzada...

- El Señor Marqués de las Hormazas, Caballero de la Real y Distinguida Orden española de Carlos III. Del Consejo de S.M. Ministro del Real Tribunal de Contaduría Mayor de Cuentas. 
- El Señor D. Manuel de Valenzuela, coronel de Ios Reales Ejércitos. Del Consejo de S.M. en el Supremo de Hacienda. Ministro de la Real Junta de Comercio, Moneda y Minas. Caballero de la Real y Distinguida Orden Española de Carlos III.

- El Señor Conde de Lerena, regidor perpetuo de la Imperial Ciudad de Toledo. Del Consejo de S.M. en el Supremo de Hacienda.

- El Señor D. Josef de Ibarra, Caballero de la Real y Distinguida Orden Española de Carlos III. Del Consejo de S.M. en el Real y Supremo de Castilla. Fiscal del Supremo de Hacienda. De la Real Junta de Comercio, Moneda y Minas.

- El Señor Marqués de Puertonuevo, Señor de la Torre Roxa y Quadra Burguesa. Del Consejo de S.M. en el Real y Supremo de Hacienda. Diputado de los Reinos por Cataluña y Mallorca. Regidor perpetuo de la Ciudad de Barcelona.

32.- AMAE. Personal español. Legajo 23. Número 1051. Expediente de D. Antonio Beramendi y Freyre.

33.- AHN. Estado. Orden de Carlos III. Expediente 1379: "Antonio Beramendi que desde el año de 1801, en que vino a esta Corte de Roma a exercer el empleo de oficial Agregado al Ministcrio de España, cerca de la Santa Sede, está gozando de la mejor lama y opinión y se ha conducido y se conduce, con la conducta más arreglada y las más loables y cristianas costumbres, como corresponde al distinguido empleo que ocupa..."

34.- MERCADER RIBA, Juan: José Bonaparte, rey de España (1808-1813). Estructura del estado español bonapartista. CSIC. Madrid, 1983. pp. 489.

35.- AMAE. Personal español. Legajo 23. Número 1051. Expediente de D. Antonio Beramendi y Freyre.

36.- AYMES, Jean-René: Los españoles en Francia 1808-1814. La deportación bajo el Primer Imperio. Siglo XXI. Madrid, 1987. pp. 124: El profesor Aymes señala que los prisioneros de Estado estaban repartidos entre el torrén de Voncennes, las fortalezas de Grilfon y Loux, las ciudadelas de Pierre-Chatel y Fenestrelle, los castillos de Ham e If. También señala que a partir de 1814, estos prisioneros de Estado lueron trasladados a Bourges o al castillo de Saumur, que es exactamente lo ocurrido con Antonio Beramendi aunque ni él ni el ministro plenipotenciario Vargas Laguna figuren en el libro del prolesor Aymes.

37.- SOLER PASCUAL, Emilio: Viajes y acción políica del Intendente Carlos Beramendi. Tesis doctoral inédita. Universidad de Alicante, junio de 1993.

38.- AGP. Papeles Reservados de Fernando VII. Tomo num. 9: Nota general de los Ministros e Individuos del Consejo de la Real Hacienda, dependientes de él, y otras oficinas, que han prestado Juranento de Fidelidad al Rey Josef Napoleón, a la Constitución, y a las Leyes, con arreglo a Decreto de 16 de febrero de 1809.

39.- AHN. Estado. Legajo 3093: Campo-Alange al Rey Ntro. Señor. Madrid, 25 de junio de 1809.

40.- AMAE. Personal español. Legajo 23. Número 1051. Expediente de D. Antonio Beramendi y Freyre.

41.- AYMES, Jean René: Los españoles en Francia 1808-1814. La deporlación bajo el Primer Imperio. Siglo XXI. Madrid, 1987. pp. 161: "Aunque la falta de detalles hace dificil establecer paralelismos entre los lugares de detención, parece que la siniestra reputación de la segunda fortaleza del Isére, Pierre-Chatel, es bien merecida. Algunos de los españoles llevan apellidos ilustres, como los hermanos Camilo y José de los Ríos, que pertenecen a la familia de los Fernán Núñez: 
¿Qué prisión! Es el receptáculo de lo más vil y lo más criminal. Y el aire demasiado sutil que se respira, las aguas del depósito que hay que beber y el poco tiempo y espacio de que se goza para pasear y que hay además que compartir con los desgraciados, todo ello resulta mortal para quien, como uno de nosotros, padece a la vez del pecho, de cálculos y de obstrucción."

42.- AYMES, Jean-René: Los españoles en Francia 1808-1814. La deportación bajo el Primer Imperio. Siglo XXI. Madrid, 1987. pp. 189: "El 31 de marzo, el número total de prisioneros de Estado, de todas las nacionalidades, se eleva a 640 , de los que 60 son cspañoles. Ignoramos qué prisioneros de Estado españoles, muy pocos, se encontraban en el castillo de If, pues todos aquellos cuyo nombre se menciona son enviados a Saumur, procedentes de Pierre-Chatel o de Vincennes. Se congregan a orillas del Loira Abad, O’Donnell, Blake, Lardizábal, "Laroca", Mina, Camino, Marco del Pont, Miranda, Romrće, Santa Cruz, Trastámara, Vargas, "d'Exaga” y algunos otros difíciles de identificar (Beramundi, Brincken, Camerling)..."

43.- AMAE. Personal cspañol. Legajo 23. Número 1051. Expediente de D. Antonio Beramendi.

44.- AMAE. Personal español. Legajo 23. Número 1051.

45.- AMAE. Personal cspañol. Legajo 23. Número 1051. Expediente de D. Antonio Beramendi y Freyre.

46.- PRADELLS NADAL, Jesús: Diplomacia y Comercio. La expansión consular española en el siglo XVIII. Universidad de Alicante e Instituto de Cultura Juan Gil-Albert. Alicantc, 1992. pp. 624.

47.- AMAE. Personal español. Legajo 23. Número 1051. Expediente de D. Antonio Beramendi y Freyre.

48.- AMAE. Personal español. Legajo 23. Número 1051. Expediente de D. Antonio Bcramendi y Frcyre.

49.- AMAE. Personal español. Legajo 23. Número 1051. Expediente de D. Antonio Beramendi y Freyre.

50.- AMAE. Personal español. Legajo 23. Número 1051. Expediente de Antonio Beramendi.

51.- GIL NOVALES, Alberto: Diccionario biográfico del Trienio liberal. Ediciones deI Museo Universal. Madrid, 1991.

52.- AN: F7 11981 y 1200.

53.- BIBLIOTECA NACIONAL. Raros 61695. BERAMENDI, Carlos de: La Inocencia trianfa al fin de la calumnia. Madrid: Imprenta de Vega y Compañía, 1820.

54.- SOLER PASCUAL, Emilio: Viajes y acción politica del Intendente Carlos Beramendi. Tesis doctoral inédita. Universidad de Alicante, junio de 1993.

55.- BIBLIOTECA NACIONAL. R-i/447. Kalendario Manual y Guía de Forasteros en Madrid. En la Imprenta Real. Años de 1815, 1816, 1817, 1818, 1819 y 1820.

56.- SOLER PASCUAL, Emilio: Viajes y acción política del Intendente Carlos Beranendi. Tesis doctoral inédita. Universidad de Alicante, junio de 1993.

57.- AMAE. Personal español. Legajo 23. Número 1051. Expediente de D. Antonio Beramendi y Freyre.

58.- AMAE. Personal español. Legajo 23. Número 1051. Expediente de D. Antonio Beramendi y Freyre.

59.- AMAE. Pcrsonal español. Legajo 23. Número 1051. Expediente de D. Antonio Beramendi y Freyre. 
60.- AMAE. Personal español. Legajo 25. Número 1085. Expediente de D. Carlos Beramendi.

61.- SOLER PASCUAL, Emilio: Viajes y acción política del Intendente Carlos Beramendi. Tesis doctoral inédita. Universidad de Alicante, junio de 1993.

62.- AGP. Expedientes personales. C-1 17/24. Expediente de D. Antonio Beramendi.

63.- AHN. Estado. Indice 1. Legajo 6235.

64.- AMAE. Personal español. Legajo 23. Número 1051. Expediente de D. Antonio Beramendi y Freyre.

65.- AMAE. Personal español. Legajo 23. Número 1051. Expediente de D. Antonio Beramendi y Freyre.

66.- AHN. Estado. Indice 1. Legajo 8364.

67.- AMAE. Personal español. Legajo 23. Número 1051. Expediente de Antonio Beramendi y Freyre.

68.- AMAE. Personal español. Legajo 23. Número 1051. Expediente de D. Antonio Beramendi y Freyre.

69.- AMAE. Personal español. Legajo 23. Número 1051. Expediente de D. Antonio Beramendi y Freyre.

70.- GIL NOVALES, Alberto: Diccionario biográfico del Trienio Liberal. Ediciones de1 Museo Universal. Madrid, 1991. pp. 82.

71.- AGP. Expedientes personales. C-1 17/24. Expediente de D. Antonio Beramendi.

72.- AGP. Expedientes personales. C-1 17/24. Expediente de D. Antonio Beramendi. 